\title{
Terawatt $x$-ray free-electron-laser optimization by transverse electron distribution shaping
}

\author{
C. Emma, ${ }^{1}$ J. Wu, ${ }^{2}$ K. Fang, ${ }^{2}$ S. Chen, ${ }^{2}$ S. Serkez, ${ }^{3}$ and C. Pellegrini ${ }^{1,2}$ \\ ${ }^{1}$ University of California, Los Angeles, California 90095, USA \\ ${ }^{2}$ Stanford Linear Accelerator Center, Menlo Park, California 94025, USA \\ ${ }^{3}$ Deutches Elektronen-Synchrotron, Hamburg 22607, Germany
}

(Received 15 May 2014; published 3 November 2014)

\begin{abstract}
We study the dependence of the peak power of a $1.5 \AA$ Terawatt (TW), tapered x-ray free-electron laser (FEL) on the transverse electron density distribution. Multidimensional optimization schemes for TW hard $\mathrm{x}$-ray free-electron lasers are applied to the cases of transversely uniform and parabolic electron beam distributions and compared to a Gaussian distribution. The optimizations are performed for a $200 \mathrm{~m}$ undulator and a resonant wavelength of $\lambda_{r}=1.5 \AA$ using the fully three-dimensional FEL particle code GENESIS. The study shows that the flatter transverse electron distributions enhance optical guiding in the tapered section of the undulator and increase the maximum radiation power from a maximum of $1.56 \mathrm{TW}$ for a transversely Gaussian beam to 2.26 TW for the parabolic case and 2.63 TW for the uniform case. Spectral data also shows a 30\%-70\% reduction in energy deposited in the sidebands for the uniform and parabolic beams compared with a Gaussian. An analysis of the transverse coherence of the radiation shows the coherence area to be much larger than the beam spotsize for all three distributions, making coherent diffraction imaging experiments possible.
\end{abstract}

DOI: 10.1103/PhysRevSTAB.17.110701

PACS numbers: 41.60.Cr, 41.60.Ap

\section{INTRODUCTION}

Self-amplified spontaneous emission $\mathrm{x}$-ray free-electron lasers (SASE X-FELs) [1-3] have given us the ability to study structures and dynamical processes at unprecedented spatiotemporal scales, with a simultaneous resolution in space and time of $1 \AA$ and 1 fs. LCLS and SACLA, the most powerful existing X-FELs, deliver diffraction limited x-ray pulses of a few to a hundred femtoseconds in the energy range of 0.25 to $10 \mathrm{keV}$ with peak power at saturation of 20-30 GW and a linewidth on the order of $10^{-3}$ [4].

$\mathrm{X}$-FELs are having a great impact on the field of bioimaging [5-7]. Research in this field will benefit from a larger number of coherent photons/pulse, a factor of 10 to 100 larger within a pulse duration of $10-20$ fs. This corresponds to a peak output power of $1 \mathrm{TW}$ or more.

It is well known that the efficiency and thus the peak power of an FEL can be increased by tapering the FEL undulator magnetic field to match the electron energy loss while preserving the synchronism condition [8]. The LCLS for example currently boosts its saturation power by a factor 2-3 to 60-70 $\mathrm{GW}$ at the undulator exit using a limited taper capacity $\Delta K / K \sim 0.8 \%$. In the case of a SASE FEL, however, the spiky characteristic of the FEL radiation [9] limits the power [10]. More recent work has shown that

Published by the American Physical Society under the terms of the Creative Commons Attribution 3.0 License. Further distribution of this work must maintain attribution to the author $(s)$ and the published article's title, journal citation, and DOI. seeding or self-seeding an FEL amplifier leads to much larger output power [11]. In light of these promising results many recent efforts have been devoted to optimize the tapered section of a self-seeded X-FEL to reach power levels of one TW or larger [12].

The analytic models developed in previous studies to obtain the optimal tapering profile have included threedimensional effects but only considered electron beams with Gaussian transverse density profile. In this paper we investigate the effects of using transversely parabolic and transversely uniform electron distributions. The results are compared to the Gaussian beam case in both single frequency and time dependent simulations using the fully three-dimensional FEL particle code GENESIS [13]. The optimizations are performed for a 200-m, self-seeded hard $\mathrm{x}$-ray tapered FEL with LCLS-II-like parameters.

The structure of the paper is as follows. In Sec. II we describe the theoretical model used to obtain the optimal tapering profile, following Ref. [12] and using results obtained from time independent GENESIS simulations. In Sec. III we discuss the time independent results for optimized taper profiles comparing the cases of Gaussian, parabolic and uniform transverse electron beam distributions. We determine the extraction efficiency for each case and examine the electron longitudinal phase space distribution and the evolution of the trapping fraction as a function of undulator distance. We then discuss the impact of time dependent effects by presenting results from multiple frequency simulations and examining the change in maximum output power, bunching and radiation size as 
well as the spectral properties of the radiation for all three electron transverse distributions. Finally in Sec. IV we study the transverse coherence properties of the output radiation from the optimized tapered X-FEL.

\section{TAPERING OPTIMIZATION METHOD}

In recent work [12] it has been pointed out that diffraction and refraction have an important effect on the peak power of X-FELs. We extend previous theories of high efficiency tapered FELs $[8,12]$ by including different electron transverse distributions. Starting from conservation of energy and applying the same assumptions as in Ref. [12], we can write the radiation power as a function of the longitudinal position in the undulator:

$$
P(z)=\frac{\pi r_{s}(z)^{2} a_{s 0}(z)^{2}}{4 Z_{0}}\left(\frac{k_{s} m_{e} c^{2}}{e}\right)^{2},
$$

where $a_{s 0}(z)=|e| A_{s}(z) / \sqrt{2} m c^{2}$ is the on-axis normalized vector potential of the radiation field for a linearly polarized undulator, $r_{s}(z)$ is the radiation beam size, $k_{s}$ is the radiation wave number and $Z_{0}$ is the free space impedance. In order to maximize this output power we must optimize the growth of the radiation field as a function of the longitudinal distance in the undulator. This can be achieved through an adiabatic decrease in the resonant energy $\gamma_{r} m c^{2}$ of the electron beam [8] where the resonant energy is given by

$$
\gamma_{r}^{2}(z)=\frac{k_{w}}{2 k_{s}}\left[1+a_{w}(z)^{2}\right]
$$

where $k_{w}=2 \pi / \lambda_{w}$ is the undulator wave number and $a_{w}(z)=|e| B_{w}(z) / \sqrt{2} k_{w} m c^{2}$ is the normalized vector potential of the undulator field, which for a tapered undulator is a function of $z$. A decrease in $\gamma_{r}$ can be obtained by varying both the undulator period and the undulator parameter; however, in this study we examine only the more convenient constant $k_{w}$ case. We apply the following taper profile, starting after the exponential regime saturation point [12]:

$$
a_{w}(z)=a_{w}\left(z_{0}\right) \times\left[1-c \times\left(z-z_{0}\right)^{d}\right],
$$

where $z_{0}$ is the location of initial saturation (see Fig. 1) and $c$ and $d$ are constants to be obtained through simulations that maximize the output radiation power. The quadrupole transverse focusing gradient $K_{q}(z)$ is also similarly optimized [12]. After the initial saturation and exponential gain regime, the FEL process is dominated by the effects of refractive guiding of the radiation by the electron beam. This can be described quantitatively by considering the complex refractive index of the beam $[12,14]$ :

$$
n=1+\frac{\omega_{p 0}^{2}}{\omega_{s}^{2}} \frac{r_{b 0}^{2}}{r_{b}^{2}} \frac{a_{w}}{2\left|a_{s}\right|}[J J]\left\langle\frac{e^{-i \Psi}}{\gamma}\right\rangle,
$$

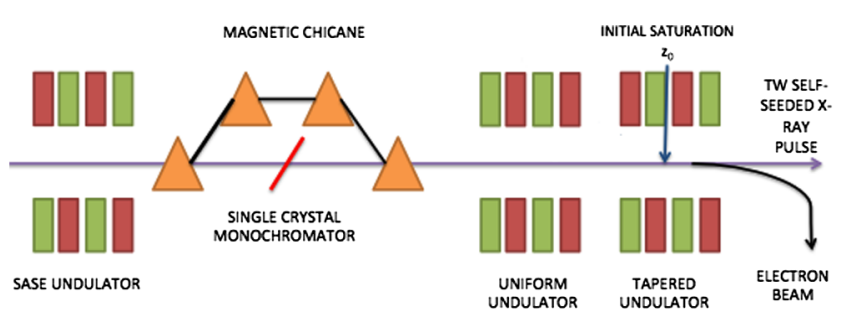

FIG. 1. Schematic representation of a tapered x-ray FEL using a self-seeding monochromator and an optimized tapered section.

where $\omega_{p}$ is the electron plasma frequency, $\omega_{s}$ is the radiation frequency and $r_{b}$ is the electron beam radius. Quantities with subscript 0 refer to initial parameters and the symbol $[J J]=$ $J_{0}(x)-J_{1}(x)$ for a planar undulator and $[J J]=1$ for a helical undulator, where $x=a_{w}^{2} / 2\left(1+a_{w}^{2}\right)$. The average term in square brackets is over the beam electrons where $\Psi$ is the electron phase relative to the ponderomotive potential.

Only electrons trapped in the ponderomotive potential well contribute to the microbunching term $\langle\exp (-i \Psi)\rangle$ and to the radiation intensity increase. It is thus important to maintain a sufficiently large microbunching throughout the tapered region as this increases the refractive index of the electron beam and boosts the coherent interaction between the electrons and the radiation [15]. It is useful at this point to consider the trapping function $F_{t}(z)$ which determines the fraction of electrons trapped in the FEL bucket along the tapered undulator:

$$
F_{t}(z)=\frac{1}{N_{e}} \int_{0}^{r_{\max }} F_{t}(r, z) f_{0}(r) 2 \pi r d r,
$$

where $f_{0}(r)$ is the transverse beam distribution and $F_{t}(r, z)$ is the local trapping fraction which is determined by the maximum and minimum phases $\Psi(r, z)$ for which particles follow stable trajectories in phase space $[8,12]$. It has been shown in [12] that the microbunching term $\langle\exp (-i \Psi)\rangle$ in Eq. (4) can be calculated by averaging the product $F_{t}(r, z) \exp \left[-i \Psi_{r}(r)\right]$ over the radial coordinate $r$. Examining Eq. (5) shows that by manipulating the initial transverse electron beam distribution $f_{0}(r)$ it is possible to maximize the trapping fraction throughout the tapered undulator. For the case of a transverse Gaussian distribution, considered thus far, the electrons in the radial tail of the beam experience a weaker electric field from those closer to the axis, and thus can become detrapped from the FEL bucket, reducing the microbunching and causing an early saturation of the power gain inside the tapered undulator. If however the transverse electron distribution is flatter, as in the parabolic or uniform cases, it is possible to trap more electrons in the bucket, increasing the bunching factor throughout the undulator and thereby extracting more power. By inspecting Eq. (4) and noting that the contribution from the Bessel functions $[J J]$ is always less than 1 for planar undulators, we can also infer that the output power will increase in a helical undulator by a factor $\mathcal{O}(1 /[J J])$. 


\section{SIMULATION RESULTS}

We first examine the model predictions with single frequency simulations using the fully three-dimensional FEL particle code GENESIS. In these simulations we optimize the taper profile and transverse quadrupole focusing for a 200-m long undulator with periodic break sections and parameters similar to those of the LCLS-II upgrade project (see Table I) [16]. After performing the multidimensional optimization, the taper profiles obtained for the three different transverse distributions are shown in Fig. 2. From these we note that the flexibility required in the undulator magnetic field is less than $\Delta K / K=10 \%$ in all three cases, making the undulator taper designs feasible. The corresponding evolution of the radiation field, power and electron beam microbunching is illustrated in Fig. 3 for the three different transverse distributions. The main result is an increase in the bunching factor for the parabolic and uniform distributions as compared to the Gaussian. This is indicative of a larger trapping fraction and consequently greater output power. Such an effect is however only marginally observed in single frequency simulations with the Gaussian beam achieving $\mathrm{P}_{\max }=2.65 \mathrm{TW}$ compared to $\mathrm{P}_{\max }=2.76 \mathrm{TW}$ for the parabolic case and $\mathrm{P}_{\max }=$ $3.03 \mathrm{TW}$ for the uniform case. What is also apparent from Fig. 3 is that the radiation size is virtually unaffected by changing the transverse beam distribution, thus the effect of optical guiding is not observed to contribute significantly when time dependent effects are not included. As predicted, the helical undulator geometry responds more favorably to the tapering optimization for all three transverse distributions, as shown in Fig. 4, reaching maximum output power over 4 TW after $200 \mathrm{~m}$.

Using the optimal undulator parameters found via the time independent simulations, we performed time dependent simulations of the three transverse distributions for 6.4 and 16 fs bunch lengths. The two bunch lengths are chosen to be around the $10 \mathrm{fs}$ range currently being considered for future experiments in single molecule imaging. The results for a resonant wavelength $\lambda_{r}=1.5 \AA$ are illustrated in Fig. 5. Time dependent effects have a major impact on the output of an optimized tapered FEL. Three-dimensional effects of the transverse electron distribution are more

TABLE I. GENESIS simulation parameters: single frequency.

\begin{tabular}{lc}
\hline \hline Parameter name & Parameter value \\
\hline Beam energy $E_{0}$ & $13.64 \mathrm{GeV}$ \\
Beam peak current $I_{p k}$ & $4000 \mathrm{~A}$ \\
Normalized emittances $\epsilon_{x, n} / \epsilon_{y, n}$ & $0.3 / 0.3 \mu \mathrm{m} \mathrm{rad}$ \\
Peak radiation power input $P_{i n}$ & $5 \mathrm{MW}$ \\
Undulator period $\lambda_{w}$ & $32 \mathrm{~mm}$ \\
Normalized undulator parameter $a_{w}$ & 2.3832 \\
Radiation wavelength $\lambda_{r}$ & $1.5 \AA$ \\
FEL parameter $\rho$ & $7.361 \times 10^{-4}$ \\
\hline \hline
\end{tabular}

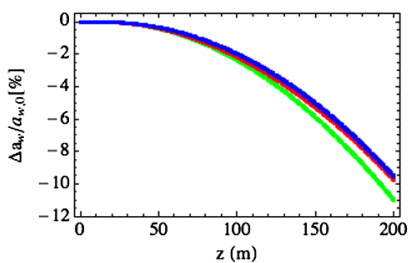

(a)

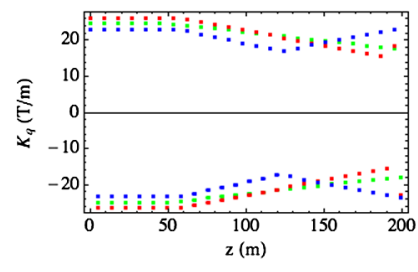

(b)
FIG. 2. Optimal taper field and quadrupole focusing profile for the Gaussian (green), parabolic (red) and uniform (blue) transverse electron distributions obtained from multidimensional optimization using GENESIS single frequency simulations.
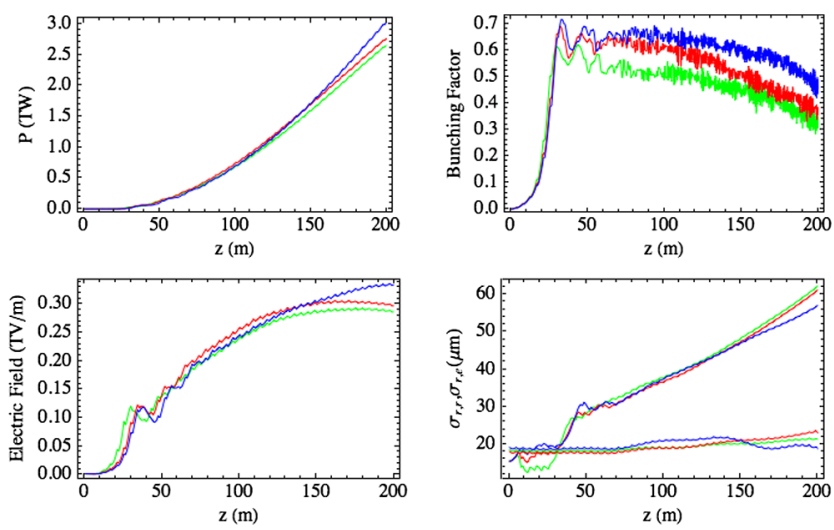

FIG. 3. Comparison of time independent results for Gaussian (green), parabolic (red) and uniform (blue) transverse beam distributions at $\lambda_{r}=1.5 \AA$ : x-ray pulse power, upper left; electric field, lower left; electron microbunching, upper right; electron and radiation beam radii, lower right.

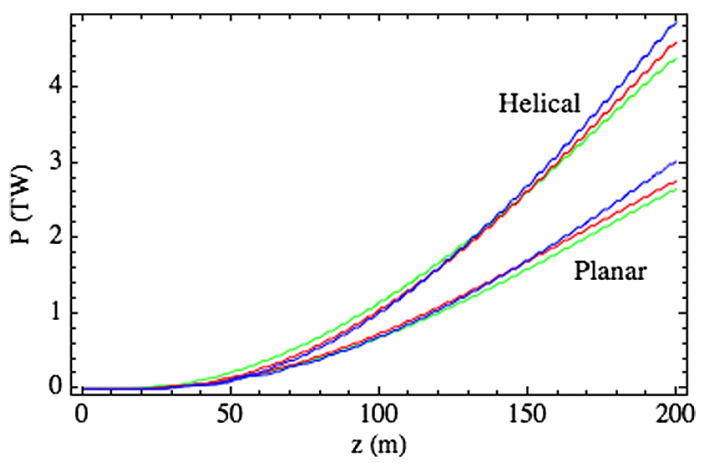

FIG. 4. Comparison of time independent results for helical and planar undulators using Gaussian (green), parabolic (red) and uniform (blue) transverse beam distributions at $\lambda_{r}=1.5 \AA$.

significant when time dependence is included. Analyzing the results shown in Fig. 5 we notice that the uniform and parabolic distributions exhibit a steady growth in output power, and a slow decrease in the bunching factor throughout the tapered undulator. On the other hand, the transversely Gaussian beam suffers a significant reduction in the bunching and power as well as an increased diffraction of 

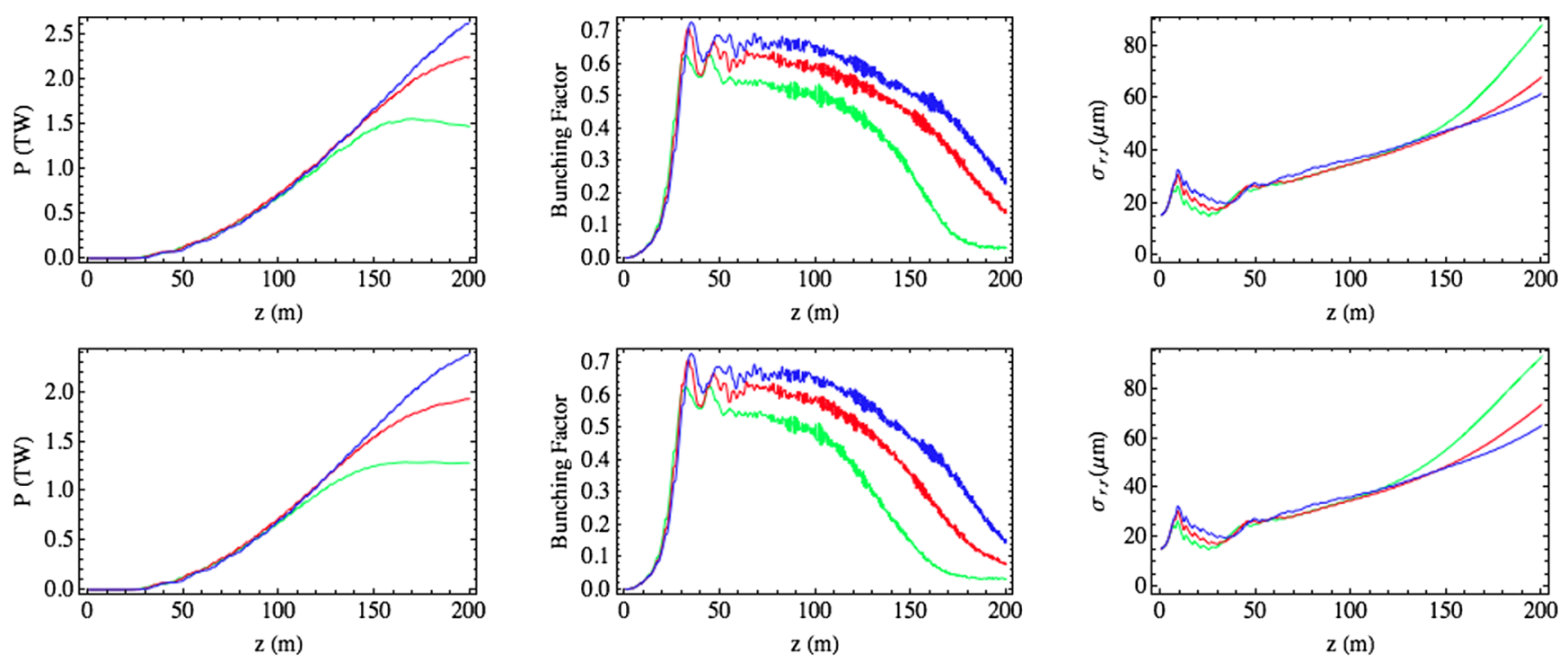

FIG. 5. Power, bunching factor and radiation size as a function of longitudinal distance for transversely Gaussian (green), parabolic (red) and uniform (blue) beams. The results are shown for a wavelength $\lambda_{r}=1.5 \AA$ and bunch lengths of $6.4 \mathrm{fs}$ (top) and $16 \mathrm{fs}$ (bottom). The optimized taper profiles are found in time independent simulations.

the radiation. Furthermore, in the time dependent case the transversely Gaussian beam shows an early saturation of the power, a result previously reported in Ref. [12]. For the three different distributions the trapping fraction is displayed in Fig. 6 and the corresponding longitudinal phase space evolution for the Gaussian beam is shown in Fig. 7. The data shows significant detrapping for the Gaussian case

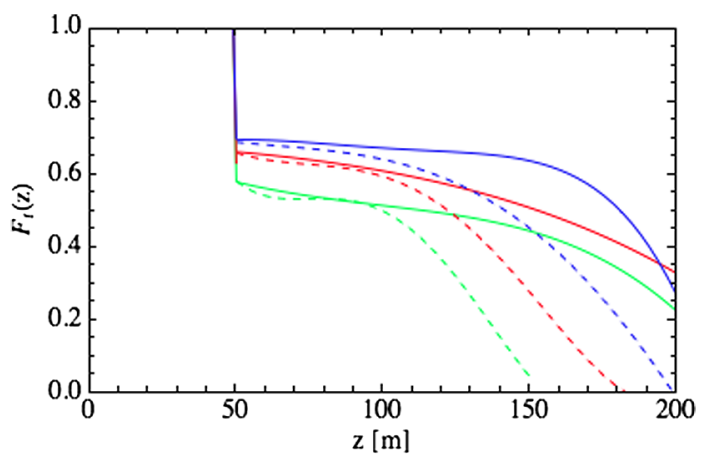

FIG. 6. Evolution of the trapping function $F_{t}(z)$ for the Gaussian (green), parabolic (red) and uniform (blue) transverse electron distributions obtained from GENESIS single frequency (solid) and time dependent (dashed) simulations.
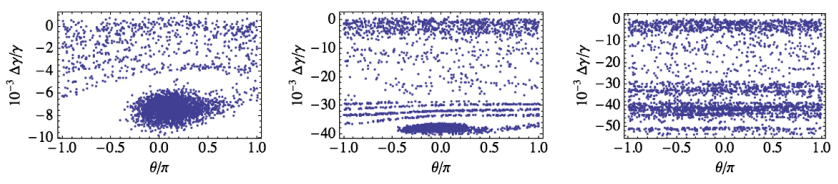

FIG. 7. Longitudinal phase space evolution for a $6.4 \mathrm{fs}$ bunch with Gaussian transverse electron distribution obtained from GENESIS time dependent simulations. The data is shown from left to right at $z=50,120,180$ meters. after $z=120 \mathrm{~m}$ which is consistent with the growth in the radiation size due to reduction in guiding observed in Fig. 5. The parabolic and uniform distributions maintain an improved trapping and guiding and this sustains the growth in output power allowing them both to reach over $2 \mathrm{TW}$ of power at $z=200 \mathrm{~m}$.

Contributing to this disparity in peak output power are the detrimental effects of the sideband instability $[17,18]$. The flatter transverse profiles of the parabolic and uniform distributions mitigate more effectively the power losses and energy deposited in the sidebands than the transversely Gaussian beam. Evidence for this is shown in Fig. 8 where observation of the spectrum at $\lambda_{r}=1.50078 \AA$ shows the Gaussian profile exhibits broader sidebands than the parabolic and uniform distributions. Examining the case for a $6.4 \mathrm{fs}$ bunch length, we integrate the power deposited in the sidebands and notice a reduction in sideband energy of

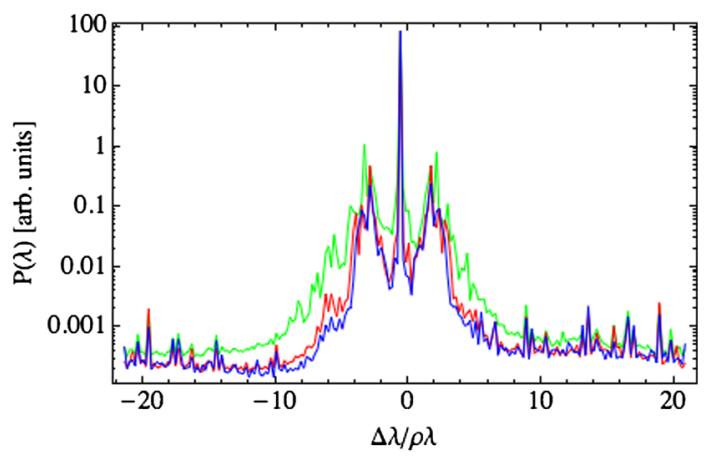

FIG. 8. Spectrum and sideband comparison for Gaussian (green) parabolic (red) and uniform (blue) transverse distributions for a 6.4 fs bunch at $\lambda_{r}=1.50078 \AA$. 
$61 \%$ for the parabolic case and $72 \%$ for the uniform beam as compared to the Gaussian. As is shown in Fig. 5 this effect, along with particle detrapping, results in a sizable difference in power output between the three transverse distributions with $\mathrm{P}_{\max }=1.56 \mathrm{TW}$ for the Gaussian beam, $\mathrm{P}_{\max }=2.26 \mathrm{TW}$ for the parabolic beam and $\mathrm{P}_{\max }=$ $2.63 \mathrm{TW}$ for the uniform beam. It is also important to note that using the optimized taper profiles for each electron distribution the extraction efficiency reaches values between $\eta=2.75 \%$ and $\eta=4.83 \%$, an order of magnitude improvement compared to state of the art $\mathrm{X}$-FELs such as the LCLS.

It is known from 3D FEL theory of fixed parameter undulators that it is possible to maximize the output power by detuning the central wavelength of the beam with respect to the resonant wavelength [19]. We examine this effect numerically in a tapered X-FEL by shifting the electron beam resonant wavelength to find the maximum of the small-signal high-gain curve. For the three transverse distributions with 6.4 fs bunch lengths, Fig. 9 shows the peak power as a function of normalized detuning $\delta=\Delta \lambda / \rho \lambda$ obtained from multiple time dependent GENESIS simulations. The maximum power for the uniform and parabolic beams is found for a redshift of $\Delta \lambda / \lambda=$ $0.34 \rho$ while the Gaussian beam peaks at $\Delta \lambda / \lambda=0.69 \rho$. The difference between the detuning can be qualitatively understood by considering an extension to the $1 \mathrm{D}$ FEL dispersion relation which includes the effect of refractive guiding. Neglecting energy spread effects we can write the dispersion relation as [20]

$$
\lambda^{3}-\left(1-\frac{\delta}{2 \rho k_{w} Z_{R}} \lambda^{2}\right)+1=0,
$$

where $\operatorname{Im}(\lambda)$ is the growth rate of the field and $Z_{R}=$ $\pi w_{0}^{2} / \lambda_{r}=2 \pi \sigma_{r, 0}^{2} / \lambda_{r}$ is the Rayleigh range of the radiation for a beam of transverse rms size $\sigma_{r, 0}$ at the waist. The 1D estimate for the maximal growth rate is then achieved for a detuning $\delta_{\max }^{(1-D)}=1 / 2 k_{w} \rho Z_{R}$ which using the value of

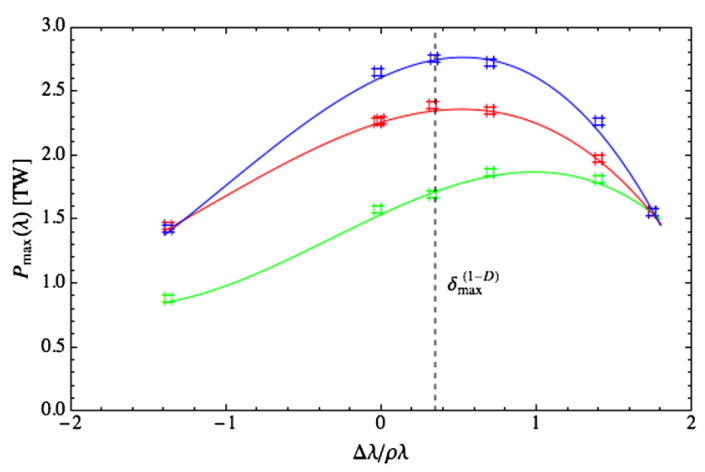

FIG. 9. Maximum radiation power as a function of detuning from resonance obtained from time dependent GENESIS XFEL simulations using transversely Gaussian (green) parabolic (red) and uniform (blue) electron distributions. $\sigma_{r, 0}=15.5 \mu \mathrm{m}$ employed in simulations yields $\delta_{\max }^{(1-D)}=$ $0.335 \rho$ (see Fig. 9). While this value is in good agreement with the uniform and parabolic results it is off by approximately a factor of 2 for the Gaussian case. The discrepancy can be understood by realizing that the increased diffraction for the Gaussian case results in a shorter Rayleigh range. Thus the maximum power is expected to occur at a larger detuning when compared with the uniform and parabolic distributions.

\section{RADIATION PROPERTIES}

We now examine the transverse coherence properties of the output radiation for the three different transverse distributions at a resonant wavelength $\lambda_{r}=1.5 \AA$ and a bunch length of $16.4 \mathrm{fs}$. We consider the mutual coherence function [21]:

$$
\Gamma(\tau)=\left\langle\vec{E}\left(\vec{r}_{1}, t\right) \vec{E}^{*}\left(\vec{r}_{2}, t\right)\right\rangle,
$$

where the electric field is sampled at two transverse locations and the angle brackets denote the ensemble average. From the mutual coherence function we define the mutual intensity function $J_{12}=\Gamma(0)$, the complex coherence factor $\mu_{12}$ and the coherence area $A_{c}$ :

$$
\mu_{12}=\frac{J_{12}}{\sqrt{J_{11} J_{22}}} \quad A_{c}=\int \mu_{12} d A
$$

In order to quantify the degree of transverse coherence we compute $\left|\mu_{12}\right|$ and the coherence area at various $z$ locations in the tapered undulator. We then compare these values to the beam spotsize and determine wether the coherence area is larger than the spotsize, a condition necessary for x-ray diffraction and imaging applications. The results at the undulator exit for the three transverse electron distributions are shown in Fig. 10. For all three distributions the spotsize $\sigma_{r}^{2}$ is $\mathcal{O}\left(10^{-3}\right) \mathrm{mm}^{2}$ while the calculated coherence area $A_{c}$ is $\mathcal{O}\left(10^{-1}\right) \mathrm{mm}^{2}$. The condition $A_{c} \gg \sigma_{r}^{2}$ is thus clearly satisfied and the radiation is sufficiently transversely coherent for x-ray diffraction and single molecule imaging experiments.

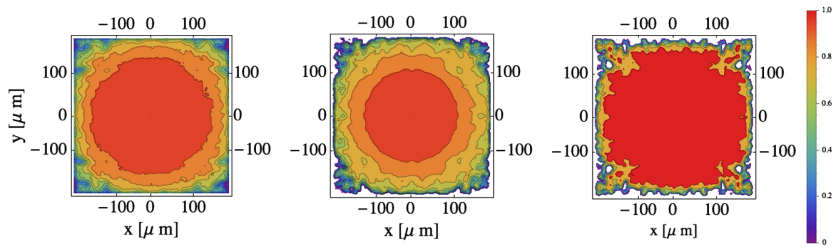

FIG. 10. Modulus of the radiation complex coherence factor at the undulator exit $(z=200 \mathrm{~m})$. Results are shown for Gaussian (left), parabolic (center) and uniform (right) transverse electron beams and a bunch length of 16.4 fs. 


\section{CONCLUSION}

We evaluated the effect of changing the transverse electron distribution in an optimized tapered free-electron laser. The performances of FELs with transverse Gaussian, parabolic or uniform beam distributions are compared. The tapering strategy applied is the multidimensional optimization method described in Ref. [12] based on maximizing the output power by improving the optical guiding and mitigating the detrimental effects of diffraction of the radiation throughout the tapered section of the undulator.

Optimizations were performed for a 200-m long undulator with break sections using the three-dimensional particle code GENESIS in time dependent and time independent simulations. Time independent results show that the effect of changing the transverse beam distribution is mostly marginal, yielding similar growth in the radiation power for the transversely Gaussian, parabolic and uniform distributions. This is not the case when multifrequency effects are taken into account in time dependent simulations, where the transverse distribution has an important impact on the FEL process affecting the trapping fraction and consequently the maximum output power. For a resonant wavelength of $\lambda_{r}=1.5 \AA$ and a bunch length of $6.4 \mathrm{fs}$ the maximal power increased from $\mathrm{P}_{\max }=1.56 \mathrm{TW}$ for the Gaussian beam, to $\mathrm{P}_{\max }=$ $2.26 \mathrm{TW}$ for a parabolic beam and $\mathrm{P}_{\max }=2.63 \mathrm{TW}$ for a uniform beam. An empirical argument related to the growth of the sideband instability, as well as a reduction in the trapping fraction, has been considered to explain this discrepancy in maximal power output. It is found that for $\lambda_{r}=1.5 \AA$ in the transversely Gaussian beam case the energy deposited in the sidebands is greater by as much as 35\% compared to the parabolic case and $47 \%$ compared to the uniform case. The effect is enhanced when one considers detuning the central beam wavelength off resonance. In this case the change between Gaussian and parabolic is $61 \%$ and between Gaussian and uniform beams is $72 \%$. For all three transverse distributions, using the optimized taper profiles, the extraction efficiency is between $\eta=2.8 \%-4.8 \%$, a factor of 20-40 improvement on current state of the art $\mathrm{X}$-FEL facilities.

A numerical study was conducted to recreate the smallsignal high-gain curve for a tapered X-FEL and compare it to established theoretical results for fixed parameter undulators [20]. Time dependent simulations show that redshifting the beam resonant wavelength by $\Delta \lambda / \lambda<2 \rho$ increases the maximum output power for all three transverse distributions. The maximum output power was obtained for a transversely flat beam at a value $\mathrm{P}_{\max }=$ $2.7 \mathrm{TW}$ at $\Delta \lambda / \lambda=0.34 \rho$.

A quantitative study of the transverse coherence properties of the radiation from optimized X-FELs has also been carried out. Results from time dependent simulations show that the transverse coherence area $A_{c}$ is larger than the beam spotsize $\sigma_{r}^{2}$ by 2 orders of magnitude for all electron beam transverse distributions, suggesting that the tapered undulator can be used in imaging and $\mathrm{x}$-ray diffraction experiments.

The study shows that transverse pulse shaping is an effective way to improve the performance and increase the output power of a tapered x-ray free-electron laser. In light of the promising results found in this study we propose to investigate methods to transversely shape the electron beam distributions, like shaping the laser pulse on the FEL injector photocathode, using suitable masks inside the beam line or introducing nonlinear elements in the electron beam transport line.

\section{ACKNOWLEDGMENTS}

This work was supported by U.S. DOE under Grant No. DE-SC0009983.

[1] R. Bonifacio, C. Pellegrini, and L. Narducci, Opt. Commun. 50, 373 (1984).

[2] J. B. Murphy and C. Pellegrini, J. Opt. Soc. Am. B 2, 259 (1985).

[3] A. Kondratenko and E. Saldin, Part. Accel. 10, 207 (1980).

[4] C. Pellegrini, in Proceedings of the 2013 North American Particle Accelerator Conference, Pasadena, California, 2010 (JACoW, 2013).

[5] M. Seibert et al., Nature (London) 470, 78 (2011).

[6] H. Chapman et al., Nature (London) 470, 73 (2011).

[7] J. S. Richardson and D. C. Richardson, Biophys. J. 106, 510 (2014).

[8] N. M. Kroll, P. L. Morton, and M. Rosenbluth, IEEE J. Quantum Electron. 17, 1436 (1981).

[9] R. Bonifacio, L. De Salvo, P. Pierini, N. Piovella, and C. Pellegrini, Phys. Rev. Lett. 73, 70 (1994).

[10] W. M. Fawley, Z. Huang, K.-J. Kim, and N. A. Vinokurov, in Proceedings of the 23rd International Free Electron Laser Conference and 8th FEL Users Workshop [Nucl. Instrum. Methods Phys. Res., Sect. A 483, 537 (2002)].

[11] W. M. Fawley, J. Frisch, Z. Huang, Y. Jiao, H.-D. Nuhn, C. Pellegrini, S. Reiche, and J. Wu, in Proceedings of the 33rd International Free Electron Laser Conference, Shanghai, China, 2011, TUOA4 (2011).

[12] Y. Jiao, J. Wu, Y. Cai, A. W. Chao, W. M. Fawley, J. Frisch, Z. Huang, H.-D. Nuhn, C. Pellegrini, and S. Reiche, Phys. Rev. ST Accel. Beams 15, 050704 (2012).

[13] S. Reiche, Nucl. Instrum. Methods Phys. Res., Sect. A 429, 243 (1999).

[14] E. T. Scharlemann, A. M. Sessler, and J. S. Wurtele, Phys. Rev. Lett. 54, 1925 (1985).

[15] D. Prosnitz, A. Szoke, and V. K. Neil, Phys. Rev. A 24, 1436 (1981).

[16] https://portal.slac.stanford.edu/sites/lcls_public/lcls_ii/ Pages/default.aspx. 
[17] T. B. Yang and R. C. Davidson, Phys. Fluids B 2, 2456 (1990).

[18] R. C. Davidson and J.S. Wurtele, Phys. Fluids 30, 557 (1987).

[19] Y. H. Chin, K.-J. Kim, and M. Xie, Phys. Rev. A 46, 6662 (1992).
[20] J. Murphy and C. Pellegrini, in Frontiers of Particle Beams, edited by M. Month and S. Turner, Lecture Notes in Physics Vol. 296 (Springer, Berlin, 1988), pp. $163-219$.

[21] J. W. Goodman, Statistical Optics (John Wiley and Sons Inc., New York, 1985). 